
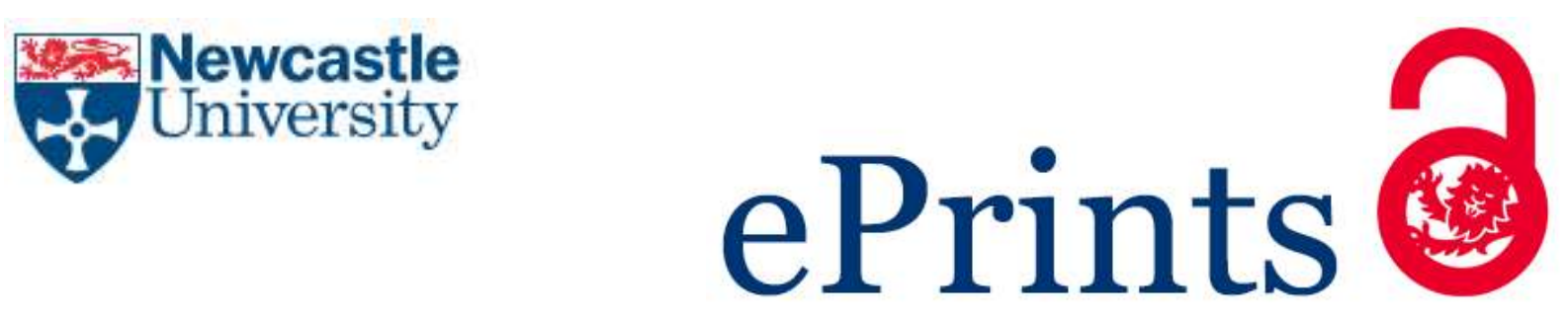

Abraham B, Davidson RI. A best space for assisted wheelchair users. British Journal of Occupational Therapy 2017

\title{
Copyright:
}

(c) 2017 SAGE Publications. This is the author's version of a work accepted for publication by SAGE Publishing

DOI link to article:

http://dx.doi.org/10.1177/0308022616662483

Date deposited:

$07 / 02 / 2017$

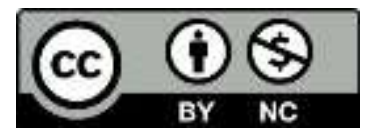

This work is licensed under a Creative Commons Attribution-NonCommercial 3.0 Unported License 


\section{A best space for assisted wheelchair users}

\begin{tabular}{|c|c|}
\hline Journal: & British Journal of Occupational Therapy \\
\hline Manuscript ID & 268-Dec-2015-RP.R1 \\
\hline Manuscript Type: & Research Paper \\
\hline Key Areas: & $\begin{array}{l}\text { Assessment < Clinical, Adult Physical < Clinical, Assistive Technology and } \\
\text { Medical Devices }\end{array}$ \\
\hline Keywords: & wheelchairs, housing, caregivers \\
\hline Abstract: & $\begin{array}{l}\text { Introduction: Occupational therapists lack manual-handling sensitive tools } \\
\text { to assist individual adaptation specifications for assisted wheelchair users, } \\
\text { for example, corridor-room turns for extra-long wheelchairs. } \\
\text { Method: Engineering based methods identified an experimental set-up. } \\
\text { This provided a useful representation of possible manoeuvres in five tasks } \\
\text { and proposed a turn difficulty order. Experienced wheelchair assistants } \\
\text { ( } \mathrm{n}=22 \text { ) selected their maximum comfortable wheelchair weight for each } \\
\text { turn. } \\
\text { Results: Some subjects (3/22) were insensitive to turning-space but all } \\
\text { other subjects (19/22) choose their lowest maximum comfortable weight } \\
\text { for the tightest turning-space and (17/19) choose their highest weights for } \\
\text { space permitting a slow turn. Mean percentage weight increased by } 30 \% \\
\text { from tight to slow turning-space. Results are statistically significant and } \\
\text { clinically important. Newcastle University Faculty and Newcastle City } \\
\text { Council Research Governance approval obtained. } \\
\text { Discussion: Experimental set-up was similar to assisting in confined } \\
\text { spaces, subjects were experienced in working in spacious environments } \\
\text { and had recent manual handling training so results are supported by good } \\
\text { manual handling practice. Assistant-size impact on easiest (highest weight) } \\
\text { turning-space is small. Results are applicable to all floor coverings and } \\
\text { wheelchair sizes but not to self-propelling wheelchair users. Results are } \\
\text { incorporated into a tool, demonstrated by case study. } \\
\text { Conclusion: tool-use specifies a best adaptation. }\end{array}$ \\
\hline
\end{tabular}




\section{Introduction}

A key occupational therapy role in adaptation services is advising how assisted wheelchair access can be restored, maintained or improved. The assistants who are assisting wheelchair users can have considerable difficulties (Roberts et al. 2011). Wheelchair users (Cooper et al. 1999) and wheelchairs (Hitchcock et al. 2006) are getting larger and heavier, homes lack space (Roberts-Hughes 2011) and manoeuvring manual vehicles in small spaces is known to be problematic (Ferreira et al. 2004). It is conceivable that assistants may find the task of assisting so difficult that they either risk injury or have their motivation to assist undermined. Occupational therapists use various methods to determine assisted wheelchair user adaptations: simulations, trials, guidelines and internal processes - just knowing the solution - acquired through practice and experience (Abraham and Johnson 2006). When these methods are sufficient they should be used. However, there will be times when the wheelchair is so long or wide, the wheelchair user so heavy, the space so confined, technical matters impose constraints, or simulations and trials are so difficult to set up that such methods are either insufficient or unsuitable. Furthermore, there are no manual-handling sensitive, evidenced based tools that guide the occupational therapist in the advice-giving process when planning adaptations for assisted wheelchair users. We present such a tool and the underpinning research.

Assisted includes assistance from friends, volunteers, family members or employees. We consider wheelchair users whose 'wheelchair' has two caster wheels at the front and two 'fixed', that is noncaster wheels, at the rear which may or may not be large enough to allow self-propelling: NHS model $8 \mathrm{~L}$ type, or not, NHS model $9 \mathrm{~L}$ type (Barrett et al. 1998). This study applies a further restriction: the assistants apply all necessary forces to the wheelchair handles; the wheelchair user makes no physical contribution.

While no suitable tools exist, assisted wheelchair users have received recent research attention: effects of cross-falls (Holloway 2011), straight-line pushing (Minns and Tracey 2010) and assistant training (Kirby et al. 2004). Recent studies of space requirements have been made (Center for Inclusive Design and Environmental Access 2010) and while these provide guidance when there are multiple, different and unknown wheelchair users, thus applicable to public buildings or new build schemes, occupational therapists in adaptations services require tools that assist recommendations for specific individuals. Assisted four-caster chair users have received recent attention (Abraham et al. 2013) but results are not applicable to assisted wheelchair users. Difficulties assisting wheelchair users in confined spaces was noted some time ago (Frank and Abel 1989) and assisted wheelchair manoeuvring has been proposed for research (van der Woude et al. 1995) but no published results exist to guide occupational therapists in individual manual-handling sensitive specifications: perhaps because researching the multiplicity of possible manoeuvres is considered difficult (Abel and Frank 1991).

Our motivations are twofold. First, to enable occupational therapists to determine a best access adaptation for assisted wheelchair users. Second, to provide occupational therapists with a means of demonstrating why a particular adaptation might be likely to succeed whereas another adaptation may not. This second motivation has three points. One, it is of value if occupational therapists can demonstrate the physical reasons why a more costly adaptation is being advised. Two, as the tool presented assists the finding of a best space for assisting a wheelchair user, it is possible that less expensive or less disruptive adaptations may be identified by its use. Three, as the tool is tailored to 
the individual wheelchair user and their assistant, there is flexibility and hence increased opportunity to make changes in a way that recognises the feelings of the home occupant as well as creating a wheelchair access space (Hawkins and Stewart 2002).

\section{Method \\ Preliminary ideas}

The tool into which the research is incorporated is an easily made card-board cut-out as shown in Table 1.1. The cut-out is scaled and the thicker rectangle represents the overall width and length of the wheelchair user and assistant: other details will be explained later in this section

Tool use includes moving the scaled cardboard cut-out on an identically scaled adaptation proposal and then determining if the individual wheelchair user and assistant would collide with the lines on the plan representing the walls. Scaled cut-out use is not new (Bumphrey 1995). However, using a cut-out without further modification is insufficient for evidence based adaptation planning for assisted wheelchair users for two reasons. First, wheelchairs with fixed rear wheels, whether large enough to self-propel or not, will not move in every direction: the area where they contact the floor will not move directly sideways. So finger movements may create impossible movements; compare four-caster equipment such as glide-about commodes which can move directly in every direction. Second, even if realistic wheelchair movements are represented on the scale plan it is not known if an assistant will find it easy or hard (definitions of easy and hard to follow) to make the movement. The research motivation is to have a manual handling sensitive tool: a scaled cardboard cut-out that moves on the plan in a way that would make the manoeuvre easy for an assistant and thus could evidence adaptation planning decisions.

The presented research is primarily of importance to occupational therapists in adaptations services. However, the necessary investigative methods for designing a tool from which adaptations can be evaluated with manual handling sensitivity are found within engineering. Table 1 provides a rigorous visual explanation of a wheelchair turn that is compatible with engineering principles.

Critically, the distances $(1100,800,308,0 \mathrm{~mm})$ for the turns shown in Table 1 were not chosen at random. Preliminary investigation (results not presented) measured each of the forces applied to each wheelchair push-handle that were required to make these movements and there are three points to note. First, these force measurements showed that the forces required to make a slower or slow turn (Table 1 figures $2-3$ ) were different to those required for a tight turn (Table 1 figure 5). However, the measurements also showed that the difference between the forces required for the slow and slower turns was relatively small. Second, it was also found that the forces changed when the turns were backwards compared with forwards: forwards is the direction the assistant or wheelchair user faces. Third, although, as expected, the forces required were relatively higher on a softer floor surface compared with a harder floor surface, the distinctions between tight and slow turns, and backwards and forwards were still present. Finding such difference is coherent with the physics since each of the different turns, tight, slow, backwards or forwards creates different combinations of wheel movements. Viewing these results in the light of Abraham (2012) suggested the possibility that assistants would find the tight turn forward hardest and the slow turn backwards easiest. An experimental set-up was created to determine if this was the case. 


\section{Subjects}

Subjects ( $n=22$ ) were social care employees who routinely assisted wheelchair users in a day centre and one was a manual handling advisor for those employees. The day centre had a hard floor and spacious accommodation throughout. Therefore, subjects were used to an environment which facilitated assisting wheelchair users. All subjects had current corporate manual handling training. There were seven males and fifteen females with mean age $47 \pm 6.8$ years.

\section{Task and task order}

Figure 1 shows a virtual image, for clarity, of the experimental set-up which has three components: first, wheelchair modified with a horizontal bar mounted above the handles: second, a stand and horizontal pole ending with a vertical pointer, placed above the horizontal bar: third, not shown, a $12.5 \mathrm{~mm}$ medium density fibre board providing a level base onto which vinyl flooring was securely adhered. The horizontal bar and the stand with vertical point ensured the set-up was identical for each subject.

The turns introduced in Table 1 (figures $2-5$ ) are two-dimensional representations of the experimental set-up. In Figure 1 the image from Table 1 figure 3 has been projected onto the floor with the white-filled directly under the vertical pointer. The researcher set the wheelchair with the vertical pointer in such a position that if the subjects turned the wheelchair while maintaining the horizontal bar under the vertical pointer, the required turn would be reproduced: the required turns being tight, quite-tight and slow turns shown in Table 1 figures $3-5$. These three turns yield five tasks as top and middle illustrations have both a backward and forward form whereas the tight turn only swops wheel movements from one side to another so mechanically the turn directions are equivalent.

Caster wheels were initially set in a consistent swivel position for each task: in what is commonly called the trail position. The trail position occurs when a very short length straight-line forward pushing occurs: $200 \mathrm{~mm}$ should cover most situations (Abraham 2012). Caster wheels are then roughly in the same orientation as the rear wheels.

All possible permutations of five tasks were determined and 22 of these permutations were selected at random.

\section{Wheelchair}

The wheelchair frame was a special size Prism T40 shower chair base modified to accept $600 \mathrm{~mm}$ diameter rear wheels with solid tyres (Urathon ${ }^{\circledR}$ ) as is typical for rear wheel shower chairs. Handles were constructed with sensors to measure forces and moments (turning effects) and mounted inline with the axis through the axle of the rear wheels (Figure 1). (Results for force and moment measurements will be presented in a separate publication.) Maximum load capacity and modifications were supervised by a registered Class 1 Rehabilitation Equipment manufacturer/modifier.

\section{Maximum comfortable weight}

Subjects selected the 'maximum comfortable weight' they could carry in the wheelchair for the five tasks. Subjects were told to select the maximum comfortable weight based on manual-handling, self-care practice and not on what they might be willing to do to assist a wheelchair user in need. Each task was initially with $70 \mathrm{~kg}$ (wheelchair and weights weighed together). The subject then choose: A) to reduce weight, B) to select the weight as 'maximum comfortable weight' or C) to try 
more weight. The invitation was made in a neutral way and the researcher repeatedly stated, 'apply manual handling principles'. Once a maximum comfortable weight was selected the task was repeated with measurements of the amount of rolling of each large wheel (angular displacement) and handle-forces. Subsequent tasks began with half the maximum comfortable weight selected for the previous task. Subjects turned the wheelchair about $30^{\circ}$ as this fully represented the physical forces that would need to be applied. This was a psycho-physical approach and it relied upon subjects who were motivated to participate (Chaffin and Andersson 2006).

Disc weights represented occupant as shown in Figure 1. Weight was distributed approximately as a typical occupant: $60 \%$ rear, $40 \%$ front (Cowan et al. 2009).

If a subject's maximum comfortable weight selection for any of the five tasks was larger or smaller than their other selection(s) then that task was deemed easy or hard, respectively, for the subject.

\section{Wheel and direction measurements}

The experimental set-up included sensors (accelerometers) to measure large wheel rolling changes. This served two purposes. First, there was nothing to stop subjects moving the wheelchair in a way that was different from the turn set by the experiment: wobbling off-course. Second, if subjects generally wobbled off-course this would indicate the additional space was required compared with the space required by following exactly as shown by the grey areas in Table 1 figure 2-5.

\section{Statistics}

There were three statistical considerations in determining a suitable test. First, normal distributions were not assumed. Second, it was necessary to allow for ties as there was nothing to stop subjects choosing the same maximum comfortable weight selection for the different tasks. Third, it was necessary to allow for missing data. The Jonckheere-Terpstra large sample approximation (Hollander 1999) meets the statistical requirements (implemented in Maple ${ }^{\circledast}$ ): this test requires a priori order of results which the preliminary study provided: there was a prior expectation that the tight turn would be associated with lower weight selections. The Kruskal-Wallis test determined associations between wobbling off-course and tasks. The sign test was used for confidence intervals.

\section{Ethics}

Subjects were social care employees but as weights were used no vulnerable adults were involved and Newcastle City Council Research Governance Level 2 and Newcastle University Faculty ethics approval was gained.

\section{Results}

Weight selections

In Figure 2 the maximum comfortable weight selections for each task is expressed as a percentage of the subjects total maximum comfortable weight selections for all tasks. In general maximum comfortable weight selections were not normally distributed (Shapiro and Wilk's W-test) so medians and $95 \%$ confidence intervals (sign test) are shown.

If there was no relationship between tight, quite-tight and slow turn variations and the weight selection then the measurements in Figure 2 would be randomly scattered, they are not. It can be seen in Figure 2 that there is a trend: the medians increase from left to right. The null hypothesis was that the selection of weights are not ordered from lowest to highest: tight (0), quite-tight forwards (+308), quite-tight backwards (-308), slow forwards (+800), slow backwards $(-800)$ : the 
numbers are the distance to the white cross as shown in Table 1 and ' $t$ ' is forwards and '-' is backwards. This was rejected: $p=0.0001$ (Jonckheere-Terpstra test). There is statistical support that the tight, quite-tight and slow turn variations and backward and forwards directions affect weight selection: tight (Table 1 figure 5 ) is associated with lowest maximum comfortable weight selections and slow backwards (Table figure 3 ) is associated with the highest maximum comfortable weight selections.

The mean percentage increases (in $\mathrm{kg}$ ) compared with the tight turn were: $6 \%$ (quite-tight forwards), 13\% (quite-tight backwards), 17\% (slow forwards) and 30\% (slow backwards): for example, one subject choose $210 \mathrm{~kg}$ for the slow turn moving backwards whereas $150 \mathrm{~kg}$ was chosen for the tight turn. This evidence shows that subjects found tight turns harder than slow ones and that the subject responded by selecting a smaller maximum comfortable weight.

Three subjects choose the same weight selections for all tasks so while different weight responses are common, 19 of 22 subjects, these are not universal: some subjects were insensitive to slow-tight turn variation and backwards and forward directions.

\section{Amount of wobble}

Subjects were asked to turn the wheelchair with a specific tightness of turn using the vertical pointer, see Figure 1, as a guide: if the vertical pointer remained on the horizontal bar then the set tightness of turn was carried out. However, there was no physical constraint to prevent the tightness of turn being different from the one set. It was therefore necessary to measure how far subjects wobbled off-course from the set tightness of turn. Figure 3 shows the wobbling off-course for each of the five tasks. If subjects had complied perfectly, all measures in Figure 3 would be close to zero, they are not. A positive value indicates the subject made a slower turn than set and negative numbers indicate a tighter turn than set. Each task in Figure 3 also has left and right pageside values: left-side values relate to wobbling off-course during the initial turn, approximately first $10^{\circ}$, wheelchair turning and right-side values are subsequent to that. Measures were generally not normally distributed and medians and $95 \%$ confidence intervals (sign test) are shown. Ranking was examined with the Kruskal-Wallis test treating all measures as positive, amount of wobbling offcourse, from low to high, was rejected ( $p=0.000$, MiniTab17) as not ordered as: slow forwards $(+800 \mathrm{~mm})$, slow backwards $(-800 \mathrm{~mm})$, tight $(0 \mathrm{~mm})$, quite-tight forwards $(+308 \mathrm{~mm})$ and quite-tight backwards $(-308 \mathrm{~mm})$. Subjects kept on-course best with quite-tight $( \pm 308)$ turns and least with the slow turn. Having both positive and negative wobbling off-course values indicates subjects varied from slow to tighter turns in one task but larger positive values show that any extra space required relates to slower turns. The keeping on-course after the first $10^{\circ}$, right-side values, is much higher. As the most wobbling off-course occurs within the initial $10^{\circ}$ it might be described as an initial wobble and it is expected that it has little effect on the space required to carry out the task since there is little overall movement of the wheelchair in the first $10^{\circ}$. The higher wobbling off-course within the first $10^{\circ}$ is consistent with the fact that the caster wheels change their direction substantially in the first $10^{\circ}$ compared to after that (Abraham 2012).

\section{Discussion}

\section{Introduction}

Four areas are covered: robustness of results under five headings, limits on application, generality of application (flooring and wheelchair type) and application through case study. 


\section{Researcher bias}

Subjects decided whether or the not maximum comfortable weight had been reached but this was partly based on how the researcher defined 'comfortable'. The researcher could have encouraged or discouraged more or less effort on one task and not another. However, the researcher was fully aware and in control of both his verbal and non-verbal behaviours and was able to ensure that subjects were unware of the researcher's internal response. Furthermore, the results are coherent with the mechanical aspects, the increasing handle-force measure from tight to slow, over which the researcher had no influence.

\section{Origin of inter-manoeuvre differences}

The results (Figure 2 show that subjects usually find slow turns easier than tight ones. Handedness and right-left upper limb strength differences are not credible explanations for these differences as the tasks involve the whole body and use of body weight is likely (Abel 1988). Also, it is possible that for the backwards tasks the weight was transferred from caster wheels to the lower roll resistance non-caster rear wheels (Abel and Frank 1991) or that subjects did not lean on the handles as much. Further reporting will be made on the latter explanation in a separate publication.

While, the biomechanical or motor skill reasons for the results have not been investigated, finding such differences coheres with related findings. Differences between sagittal (forward-backwards) strength ability and non-sagittal strength have been identified (Kumar 1995). Strength is affected by the direction in which the strength is used. The results also cohere with assistant responses to fourcaster chairs (Abraham et al. 2013).

\section{Tight to slow turns and wobbling off-course}

Figure 3 shows that the slow turns have much more wobbling off-course than the tight turns. Nevertheless, there are three reasons for concluding that maximum comfortable weight selections were not a result of wobbling off-course. First, if subjects were equally free to vary their wobbling across all five tasks why did they not do so? The wobbling off-course variation between tasks suggests there are keeping on-course difficulties with slow turns rather than that subjects wobbled off-course to choose larger maximum comfortable weight selections. Second, the quite-tight turns have higher keeping on-course and higher maximum comfortable weight selections than the tight turns. Third, intra-subject examination shows that the subjects $(n=3)$ who did not vary maximum comfortable weight selections have the same pattern of wobbling off-course variation as other subjects. Wobbling off-course does not explain the weight selection results.

\section{Non-experimental assisted wheelchair use}

The experimental set-up was artificial. Ordinarily assistants do not use a vertical pointer set above a horizontal bar when turning. However, in real use, for example, the wheelchair user's toe is kept in view to ensure no toe-wall contact so while the location of the critical object to view changes, the task is not materially different.

The second artificiality was that subjects were asked to maintain a specific measurement of tight to slow turning whereas in ordinary use assistants may, if space permits, vary the turn. However, the slow to tight turning variation is limited even when space is abundant. If Table 1.2-5. is examined it will be seen that the white-filled cross is always on the axis through the rear wheel axle and if other turns were drawn they would show the same feature. But, where space is not in abundance, a motivational reason for this research, the turning variation is severely limited by avoiding wall and fixture collisions: this is what occurred with the experimental set-up. Subjects attempted to maintain 
turning tightness variation but to some extent it varied and they wobbled off-course (Figure 3). The apparent experimental artificiality is a good representation of confined spaces.

\section{Good manual handling practice}

By virtue of their experience assisting wheelchair users in a spacious environment and their current manual handling training, the subjects should be viewed as having an authoritative opinion. Such an opinion ought to guide practice until such time as different evidence is available from equally well informed subjects.

\section{Limitations}

The results should not be applied to self-propelling wheelchair users as the occupant applies forces to the wheels and not the handles. Wheelchairs with non-caster wheels at the front and caster wheels at the rear require separate consideration. The results cannot be applied to tasks of long duration such as going round a sports field.

\section{Flooring and wheelchair type}

Flooring and wheelchairs will vary from the experiment, the latter in width and length, wheel and tyre type, position and handle type, handle-position in relation to the axis through the rear wheel axle. However, the underlying mechanical effect which requires different handle-forces for different combinations of turn tightness and clock directions and the varying response of the assistant will remain. So while the maximum comfortable weight in actual terms, the number of kilograms, would vary, definitely lowering on softer floorings and probably lowering as the rear wheel to caster wheel distance lengthens (Abraham 2012), the qualitative differences between slow and tight, and backwards and forwards will not disappear.

If the combined wheelchair and user weight is low, floor-covering is hard and assisting is easy, there will be no disadvantage to having a tight turn. However, where manual handling difficulties are a concern the results are an important part of a best space determination: part not whole as it may still be necessary to replace thick carpets, have a lower motion resistance wheelchair or assistants unaffected by the task particularly for assistants who are insensitive to tightness of turn.

\section{Case study}

This work seeks to provide occupational therapists with an evidence based manual-handling sensitive tool for determining a best adaptation for assisted wheelchair users. There will be situations where therapists are appropriately confident of success without recourse to such a tool. However, there will also be interventions where determining the adaptation is much more difficult. The case study illustrates this. The case study is compiled from more than one (first author) interventions.

While in hospital an assisted wheelchair user had a leg-raising extension specified for her $8 \mathrm{~L}$ type wheelchair resulting in an approximately $270 \mathrm{~mm}$ increased wheelchair length. The adaptation was being planned in advance of discharge. Assistance with wheelchair movement was to be from a care provider so multiple unknown assistants would assist access between the building-exterior 
(external) via hall and a 'room' as shown in part-plan Figure 4. 1. To avoid visual clutter neither doors nor architectural measurements are shown. The wheelchair user was $100 \mathrm{~kg}$ ( $16 \mathrm{stone}$ ) and manual handling concerns regarding wheelchair assistance had been expressed by the care provider who had provided assistance prior to hospital admission. The occupational therapy task was to specify doorway size, position and corridor width.

The tool, a simple cardboard scale cut-out as described in the preliminary ideas (Table 1 figure 1 ) is used and details of tool-making are elaborated in Table 2 .

Measurement ' $A$ ', Table 2, is determined by wheelchair and wheelchair user anthropometrics: it accommodates the $270 \mathrm{~mm}$ increase to the $8 \mathrm{~L}$ wheelchair length. Measurement ' $\mathrm{B}$ ' is dependent upon wheelchair $(300 \mathrm{~mm})$ and assistant size: $400 \mathrm{~mm}$ is allowed for the assistant and while this research did not measure assistants, the uncertainty will be shown later in the case study to be manageable. Wheelchair width is $616 \mathrm{~mm}$. The turning points (see Table 1 figures 2 and 3 ) for slower and slow turns are indicated on the tool with white-filled crosses.

Figure 4.1 shows the initial layout and Figure 4.2 shows an assistant, wheelchair and occupant positioned ready for a backwards turn into the room having come from the external. Figure 4.2 evidences the problem using the slow turn backwards - the relevant cross is black-filled - since 'wall $A^{\prime}$ is clearly a barrier to turning: the intuitive sense of there being a problem is visually represented in accordance with the physics. The thick black curves show the most outer and most inner areas traversed. The left bottom corner of the tool in Figure 4.2 representing the corner of the footplate, the left-top corner of the tool representing the rearmost part of the assistant and left elbow of the wheelchair user. The thick black dashed curves show how these area perimeters change if the assistant wobbles off-course and uses the slower turn. It can be seen that wobbling off-course, the slower turn, actually results in less corridor width but requires a change in door-opening position.

The evidenced turn in Figure 4.2 leads to 'Proposal 1' shown in Figure 4.3 where the 'wall A' barrier is removed by creating a new doorway. However, as some assistants may find that their easiest turn is slow forwards the exercise is repeated and Proposal 1 is subject to the same examination using a slow forward turn as shown in Figure 4.4 : having come from the external the assistant, wheelchair and occupant positioned to turn into the room moving forwards. Figure 4.4 shows that Proposal 1 provides more door opening space than essential for the forwards slow turn of Figure 4.4. This leads to Proposal 2 in Figure 4 .5. (One possible door-type is double inward opening doors. Doors can be managed by the assistant.) As the research has found that some assistants found a quite-tight turn easiest the process shown in Figure 4 should be repeated beginning with Proposal 2. Finally, the journey in the reverse direction, starting from the 'room' and moving to 'external' should also be considered. The case study has assumed the wheelchair user had a preference for entering the property forward-facing but the tool can be used for rearward-facing entry. For brevity these additional steps are not included here. The first author has found that after some practice, tool creation and use takes minimal time and the task only becomes time-consuming when a neat record of all investigated movements is required.

The illustrated Proposal 2 in Figure 4.5 is one idea: there are others. The wheelchair starting position in Figure 4.2 could be nearer the external or further away from external in Figure 4.4 or 'wall A' could be moved to page-right. There may be technical aspects such as electrical cabling or wheelchair user or family preferences that make one idea more or less attractive than another. The tool provides a way of evaluating whichever ideas are presented: adaptation planning is complex. 
It would require further research to determine how the space required is affected by assistant size and positioning. However, the assistant will grasp both wheelchair handles so there is a limit to how much lateral variation is possible. Furthermore, it was the researcher's impression that none of the subjects adopted anything but a fairly upright walking stance and this is consistent with the task since extreme effort was not being sought. While assistant distance from wheelchair has a direct effect on the opening size, the area of potential collision is identified by the tool. Additionally, the assistant is at some distance from any obstruction for the hardest part of the task in Figure 4.2 and Figure 4. 4, beginning movement, so optimal positioning may not be as important where assistantwall collision would occur. It is also to be noticed that using a simple rectangular shape for the tool increases the space required since the assistant will not be rectangular. In Figure 4.4 the door will be open on entry and the only intrusion into an assistant's preferred space would be at the corner of the wall at the start of the stairs.

The research and resulting tool are a guide to adaptation decision making. Nevertheless, knowledge of tool-use incorporates good manual-handling practices. So, for the case study illustration, if after completing the adaptation, the care provider reported that some carers where having difficulty, the occupational therapist is in a position to advise that the adaptation allows the room to be entered both forwards and backwards which may resolve difficulties.

Beyond the specifics of the case study, if only a single assistant is involved then the results and tool can be used to design for a single individual. If the reader wishes to create a template for a chair of a substantially different width, for example an $816 \mathrm{~mm}$ wide chair, then all the measurements in Table 2 and Figure 4 are adjusted as follows. Add half the width difference from the experimental chair of $616 \mathrm{~mm}$ : half of $816-616 \mathrm{~mm}$ is $100 \mathrm{~mm}$, so for the quit-tight turn $308+100 \mathrm{~mm}$ is $408 \mathrm{~mm}$, for the slow turn $800+100 \mathrm{~mm}$ is $900 \mathrm{~mm}$ and $1100+100 \mathrm{~mm}$ is $1200 \mathrm{~mm}$ with each of the three measurements $(408,900$ and $1200 \mathrm{~mm})$ being the measurements in Table 2 but adjusted for the $816 \mathrm{~mm}$ wide chair. The tight turn is always at the midpoint of the axle between the non-caster rear wheels and is therefore $0 \mathrm{~mm}$ for all chair widths.

\section{Conclusion}

The tool represents the physical motion of wheelchairs and incorporates a rigorous research based manual handling sensitivity. Using the evidence based tool will predict a best adaptation for assisted wheelchair users even when space is compromised or the wheelchair and occupant are large. Learning the method requires a time investment but it can be used when other methods of determination are insufficient and once learnt, application is rapid. Using the tool, occupational therapists can both evaluate adaptation proposals and create adaptation proposals. Additionally, the process can clearly show what parts of the building need and do not need to be adapted. Not least, the tool makes it transparent why one adaptation may be preferred to another.

"This work was supported by the United Kingdom Occupational Therapy Research Foundation" 
Welford Day Centre, Newcastle upon Tyne

Technical Aids Service, Freeman Hospital, NHS England North Medical Physics

Bill Lowes (virtual wheelchair image)

Abel, EW (1988) A Biomechanical study of the manual pushing of vehicles. PhD, University of Dundee, Dundee.

Abel, EW and Frank, TG (1991) The design of attendant propelled wheelchairs. Prosthet Orthot Int, 15(1). 38-45.

Abraham, B, Davidson, RI and Johnson, GR (2013) Adaptation planning guideline and constrained outline for shower chairs and other four-caster vehicles. British Journal of Occupational Therapy, 76(10). 443-469.

Abraham, B and Johnson, G (2006) Constrained outlines: a method for creating access guidelines for individual wheelchair users. British Journal of Occupational Therapy, 69(8). 379-385.

Abraham, BB (2012) Forces applied and space required relationship for four caster vehicle manoeuvres. Ph D, Newcastle University, Newcastle upon Tyne.

Abraham, BB and Davidson, RI ('in press') Myths, legends and wheelchair turning circles: assisted wheelchair users turn in spirals In Paper Presented to the The College of Occupational Therapists 40th Annual Conference and Exhibition and the Specialist Section - Trauma \& Orthopaedics Annual Conference, Harrogate, UK.

Barrett, J, Baxendale, KM and Kelsall, ADC, G M (1998) Manual wheelchairs a practical guide, 1 ed., Oxford: Disability Information Trust.

Bumphrey, EE (1995) Community practice, a text for occupational therapists and others involved in community care, London: Prentice Hall Harvester Wheeatsheaf. 
Center for Inclusive Design and Environmental Access (2010) Anthropometry of Wheeled Mobility Project: The State University of New York.

Chaffin, DB and Andersson, GBJM, Bernard. J. (2006) Occupational Biomechanics, 4 ed., New Jersey: Wiley-Interscience.

Cheney, W and Kincaid, D (1994) Numerical mathematics and computing, Pacific Grove, California, USA: Brooks/Cole.

Cooper, RA, O'Connor, TJ, Gonzalez, JP, Boninger, ML and Rentschler, A (1999) Augmentation of the $100 \mathrm{~kg}$ ISO wheelchair test dummy to accommodate higher mass: a technical note. Journal of Rehabilitation and Research Development, 36(1). 48-54.

Cowan, RE, Nash, MS, Collinger, JL, Koontz, AM and Boninger, ML (2009) Impact of Surface Type, Wheelchair Weight, and Axle Position on Wheelchair Propulsion by Novice Older Adults. Archives of Physical Medicine and Rehabilitation, 90(7). 1076-1083.

Craig, JJ (1986) Introduction to robotics: mechanics and control, 2 ed., Reading, Massachusetts, USA: Addison-Wesley.

Ferreira, JJ, Boocock, MG and Gray, MI (2004) Review of the risks associated with pushing and pulling heavy loads Health and Safety Executive.

Frank, TG and Abel, EW (1989) Measurement of the turning, rolling and obstacle resistance of wheelchair castor wheels. Journal of Biomedical Engineering, 11(November). 462-466.

Hawkins, R and Stewart, S (2002) Changing rooms: the impact of adaptations on the meaning of home for a disabled person and the role of the occupational therapists in the process. British Journal of Occupational Therapy, 65(2). 81-87.

Hitchcock, D, Hussey, M, Burchill, S and Galley, M (2006) A Survey of occupied Wheelchairs and Scooters: Mobility and Inclusion Unit of the Department for Transport.

Hollander, MW, Douglas A. (1999) Nonparametric statistical methods, Wiley series in probability and statistics, 2 ed., New York: John Wiley \& Sons inc.

Holloway, C (2011) The Effect of Footway Crossfall Gradient on Wheelchair Accessibility. PhD, University College London, London.

Johnson, B and Aylor, J (1985) Dynamic modeling of an electric wheelchair. IEEE Transactions on Industry Applications, vol. IA-21(no. 5). 1284-1293.

Kirby, RL, Mifflen, NJ, Thibault, DL, Smith, C, Best, KL, Thompson, KJ and MacLeod, DA (2004) The manual wheelchair-handling skills of caregivers and the effect of training. Archives of Physical Medicine and Rehabilitation, 82(12). 2011-2019.

Koontz, AM, Cooper, RA, Boninger, ML, Yang, Y, Impink, BG and van der Woude, LHV (2005) A kinetic analysis of manual wheelchair propulsion during start-up on select indoor and outdoor surfaces Journal of Rehabilitation Research and Development, 42(4). 447-458.

Major, RE (1990) Some aspects of wheeled geometry relating to manually propelled wheelchairs. Physiotherapy, 76(10). 663-665.

Minns, J and Tracey, S (2010) Wheelchair pushing forces over a vinyl and a new shock -absorbing flooring. British Journal of Occupational Therapy, 74(1). 41-43. 
Roberts-Hughes, R (2011) The Case for Space: the size of England's new homes, London: RIBA.

Roberts, JY, Hannah, Andrew, K, McAlpin, A and Hogg, J (2011) Keeping the Wheels Turning: a research project investigating the needs of carers supporting people who use wheelchairs. Dundee.

van der Woude, LHV, van Koningsbruggen, CM, Kroes, AL and Kingma, I (1995) Effect of push handle height on net moments and forces on the musculosketal system during standardised wheelchair pushing tasks. Prosthet Orthot Int, 19(3). 


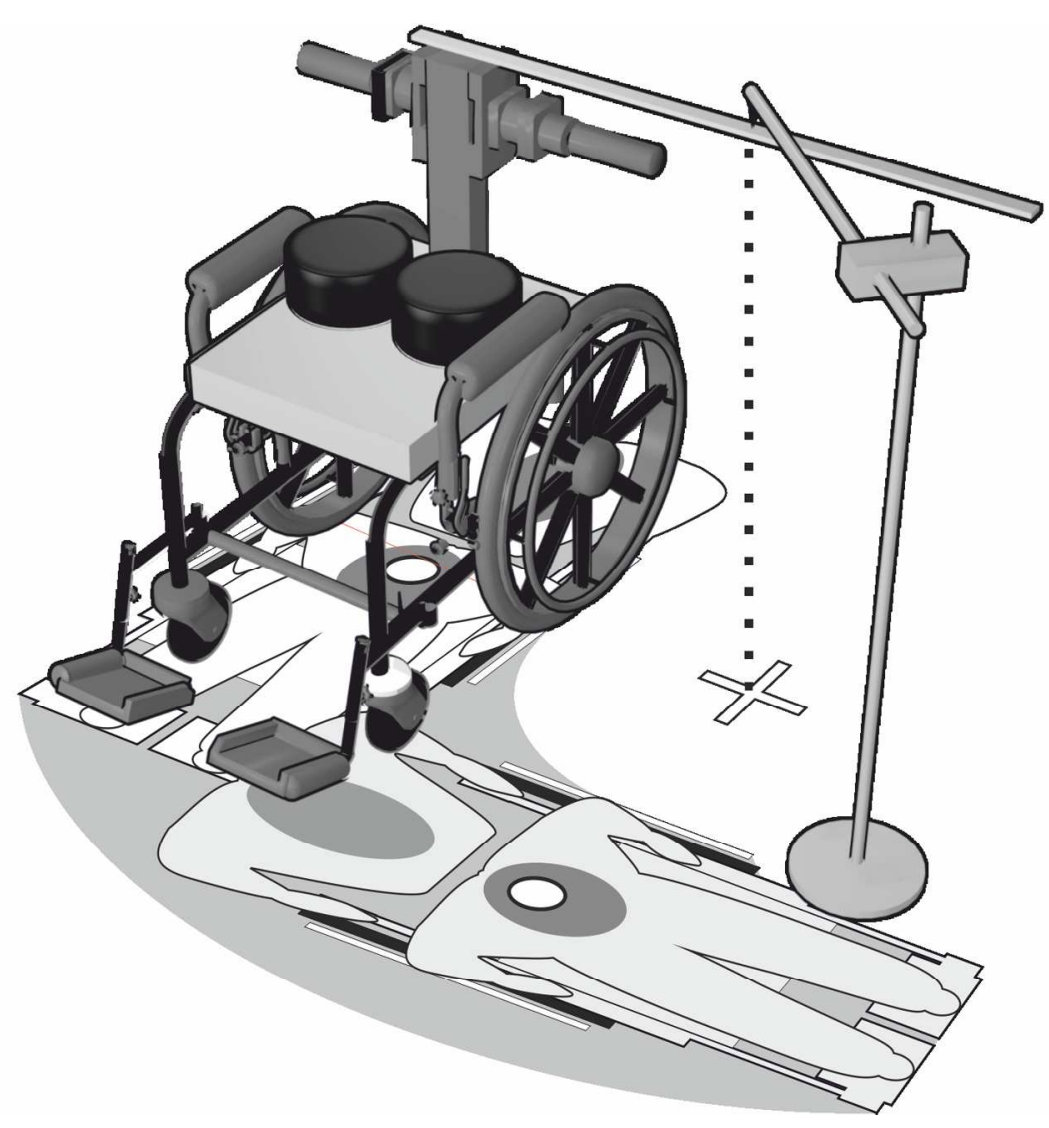

Figure 1 shows, in virtual form the experimental wheelchair with weights representing the occupant, and horizontal bar and vertical pointer 


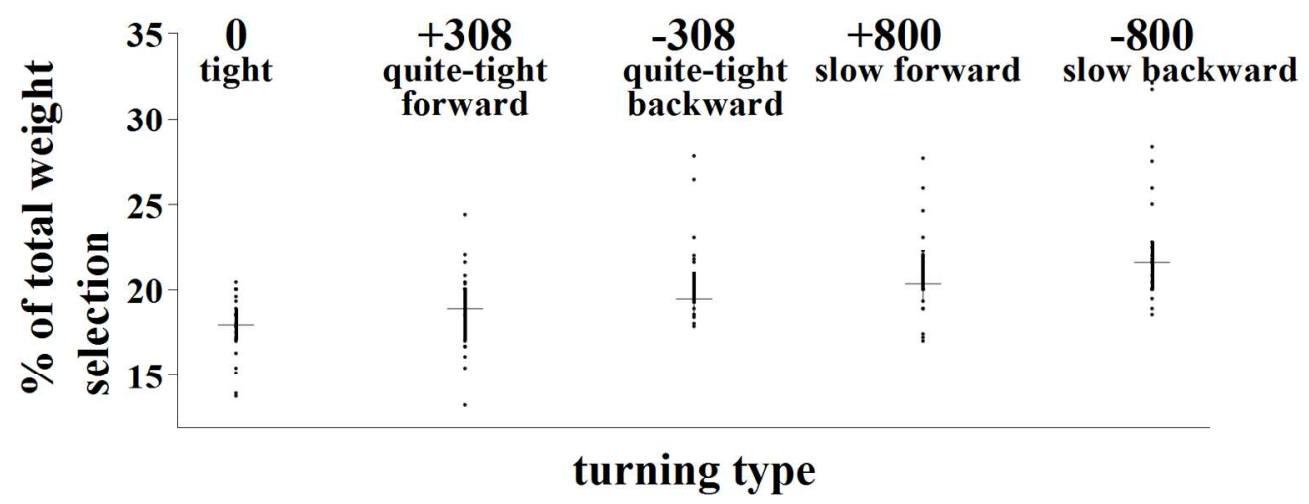

Figure 2 Shows the weight selections against the turning type $650 \times 249 \mathrm{~mm}(72 \times 72 \mathrm{DPI})$ 


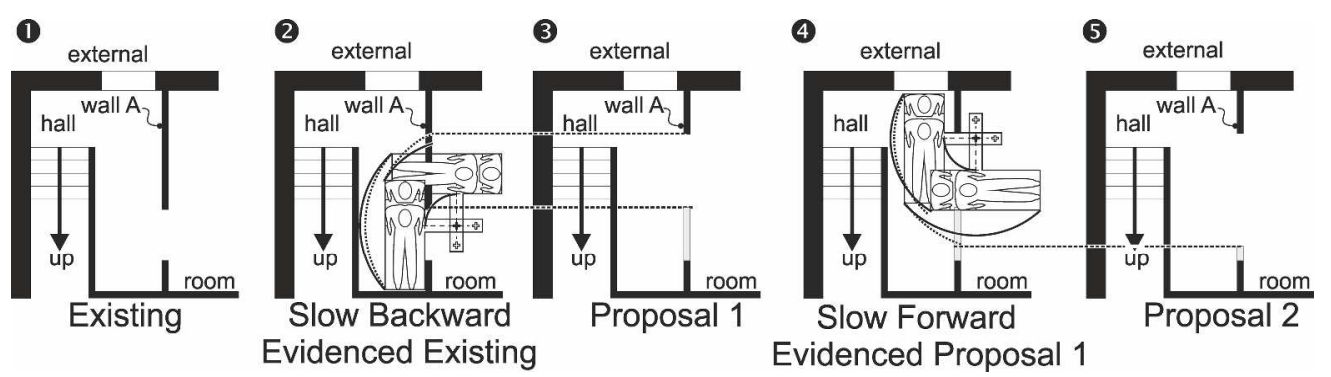

Figure 4 shows the initial situation (1), a first application of the tool for a clockwise turn (2), the resulting proposal (3), a second application of the tool for an anticlockwise turn (4) and further proposal. 


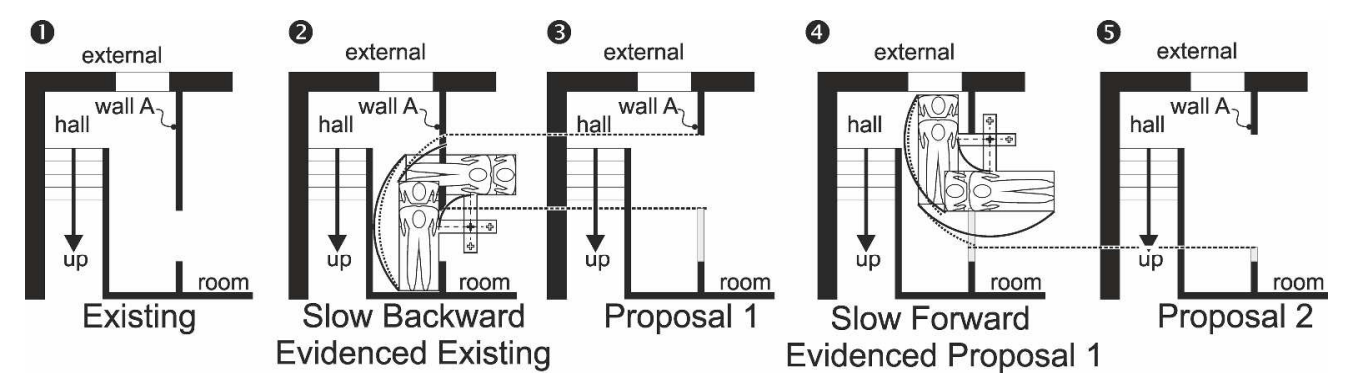

Figure 4 shows the initial situation (1), a first application of the tool for a clockwise turn (2), the resulting proposal (3), a second application of the tool for an anticlockwise turn (4) and further proposal. 


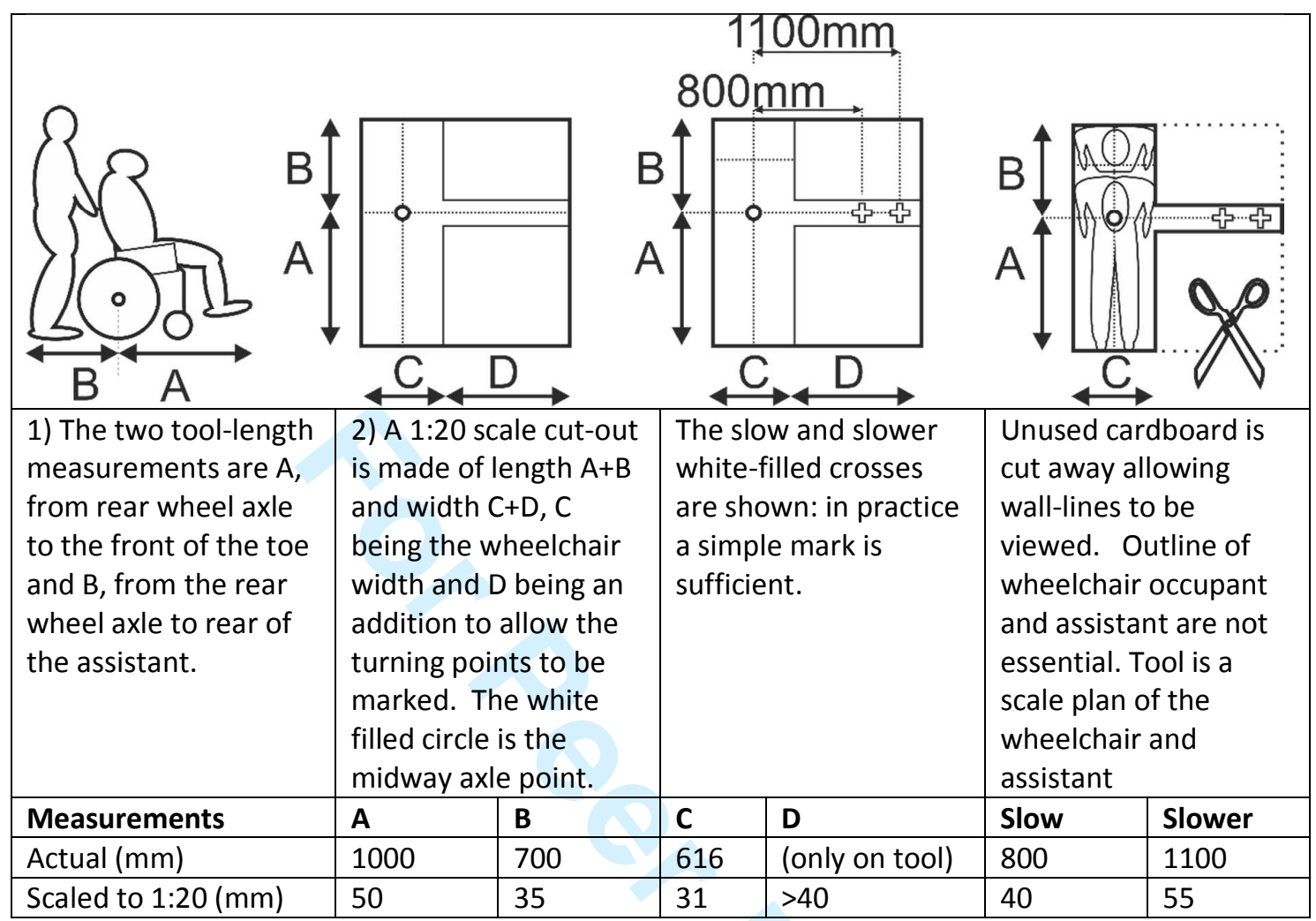

